Mon. Not. R. Astron. Soc. 000,1 1-? (2002) Printed 17 November $2017 \quad$ (MN LATEX style file v2.2)

\title{
Extreme Quiescent Variability of the Transient Neutron Star Low-mass X-ray Binary EXO 1745-248 in Terzan 5
}

\author{
- L. E. Rivera Sandoval ${ }^{1,2 \star}$ R. Wijnands ${ }^{1}$, N. Degenaar ${ }^{1}$, Y. Cavecchi ${ }^{3,4}$, C. O. Heinke ${ }^{5}$, \\ E. M. Cackett ${ }^{6}$, J. Homan ${ }^{7,8,9}$, D. Altamirano ${ }^{10}$, A. Bahramian ${ }^{11}$, G. R. Sivakoff ${ }^{5}$, \\ J. M. Miller ${ }^{12}$ \& A. S. Parikh ${ }^{1}$ \\ 1 Anton Pannekoek Institute for Astronomy, University of Amsterdam, Science Park 904, 1098 XH Amsterdam, The Netherlands \\ 2 Department of Physics, Box 41051, Science Building, Texas Tech University, Lubbock, TX 79409-1051, USA \\ 3 Department of Astrophysical Sciences, Princeton University, Peyton Hall, Princeton, NJ 08544, USA \\ ${ }^{4}$ Mathematical Sciences and STAG Research Centre, University of Southampton, SO17 1BJ, UK \\ 5 Department of Physics, University of Alberta, CCIS 4-183, Edmonton, AB 435 T6G 2E1, Canada \\ ${ }^{6}$ Department of Physics \& Astronomy, Wayne State University, 666 W. Hancock St, Detroit, MI 48201, USA \\ 7 MIT Kavli Institute for Astrophysics and Space Research, 77 Massachusetts Avenue 37-582D, Cambridge, MA 02139, USA \\ 8 SRON, Netherlands Institute for Space Research, Sorbonnelaan 2, 3584 CA Utrecht, The Netherlands \\ 9 Eureka Scientific, Inc., 2452 Delmer Street, Oakland, CA 94602, USA \\ 10 Physics and Astronomy, University of Southampton, Southampton SO17 1BJ, UK \\ 11 Department of Physics and Astronomy, Michigan State University, East Lansing, MI, USA \\ 12 Department of Astronomy, University of Michigan, 1085 South University Ave, Ann Arbor, MI 48109-1107, USA
}

17 November 2017

\begin{abstract}
EXO $1745-248$ is a transient neutron-star low-mass X-ray binary that resides in the globular cluster Terzan 5 . We studied the transient during its quiescent state using 18 Chandra observations of the cluster acquired between 2003 and 2016. We found an extremely variable source, with a luminosity variation in the $0.5-10 \mathrm{keV}$ energy range of $\sim 3$ orders of magnitude (between $3 \times 10^{31} \mathrm{erg} \mathrm{s}^{-1}$ and $2 \times 10^{34} \mathrm{erg} \mathrm{s}^{-1}$ ) on time scales from years down to only a few days. Using an absorbed power-law model to fit its quiescent spectra, we obtained a typical photon index of $\sim 1.4$. This indicates that the source is very hard throughout the full X-ray luminosity range. Only at the highest luminosity the spectral fit requires an additional soft component. These quiescent properties are likely related to strong variability in the low-level accretion rate in the system. However, its behavior is strikingly different from that observed for other neutron star transients that are thought to still accrete in quiescence. We compare our results to these systems. We also discuss similarities and differences between our target and the transitional millisecond pulsar IGR J18245-2452, which also has hard spectra and strong variability during quiescence.
\end{abstract}

Key words: X-ray binaries - globular clusters: individual: Terzan 5.

\section{INTRODUCTION}

A low mass X-ray binary (LMXB) is a system in which a low mass star (typically $\lesssim 1 \mathrm{M}_{\odot}$ ) has filled its Roche lobe and transfers mass to a neutron star (NS) or a black hole (BH). The type of accretor can often be identified as being a NS when thermonuclear X-ray bursts or X-ray pulsations

\footnotetext{
* E-mail:liliana.rivera@ttu.edu
}

are detected since these phenomena require the presence of a solid surface and/or a magnetic field.

Transient LMXBs are systems that undergo episodes of active accretion (called outburst) after long periods (lasting up to decades) of quiescence. These outburst episodes typically last at most several months. They are thought to be caused by instabilities in the accretion disc (see the review by Lasota 2001) and can reach peak X-ray luminosities of $L_{X} \sim 10^{35-39} \mathrm{erg} \mathrm{s}^{-1}$. During the quiescence period no or 
only little accretion of matter occurs and the LMXBs are observed at X-ray luminosities of only $10^{30-34} \mathrm{erg} \mathrm{s}^{-1}$.

The X-ray spectra of the quiescent systems that harbour BHs (see e.g. Kong et al. 2002, Hameury et al. 2003 Tomsick et al. 2003 Plotkin et al.|2013) and the spectra of a subset of the NS systems (e.g. Campana et al. 2002 Wijnands et al.|2005, Heinke et al. 2007, 2009a||Degenaar \& Wijnands 2012: Degenaar et al. 2012a) can be described by a hard component, which is typically modeled with a powerlaw model. In the BH systems, this component has to come from some form of accretion onto the BH (see Plotkin et al. 2013 , and references therein for a detailed discussion), but for the NS systems the origin of this hard component is not clear. It could be related to accretion as well or it could be due to physical processes that involves the NS magnetic field (see Degenaar et al. 2012b, for an in-depth discussion).

Although a significant number of quiescent NS systems have hard spectra, the majority of the NS systems either have pure soft, thermal quiescent spectra (typically described by a neutron-star atmosphere model, e.g. Wijnands et al. 2003, 2004 Degenaar \& Wijnands 2011; Lowell et al. 2012 Homan et al. 2014) or a two component spectrum in which the thermal and the non-thermal components both contribute significantly (e.g., Asai et al. 1996, 1998; in't Zand et al. 2001, Rutledge et al. 2001, 2002, Tomsick et al. 2004 Cackett et al. 2005). When the spectra are strongly dominated by the soft component, it is typically thought that the emission originates from the cooling emission of a NS that has been heated due to the accretion of matter (see the review by Wijnands et al. 2017). When a strong hard component is present as well, the origin of both components is less well understood. Although accretion onto the NS surface had been considered as the reason for the soft component (Campana et al. 1998), only recently strong evidence emerged that indeed both components arise from accretion onto the NS, with the soft component coming directly from the surface (due to potential energy release when the matter hits the surface) and the hard component originating from the boundary layer (through Bremsstrahlung; Chakrabarty et al. 2014, D'Angelo et al. 2015). Wijnands et al. (2015) suggested that if both components contribute $\sim 50 \%$ to the unabsorbed $0.5-10 \mathrm{keV}$ flux, both components likely originate due to accretion.

Transient LMXBs have been identified in different environments of our Galaxy. Most are located in the disc of the Galaxy, but some of them reside in globular clusters (GCs), such as Terzan 5. This GC is located at $5.5 \pm 0.9 \mathrm{kpc}$ (Ortolani et al. 2007) in the direction towards the bulge of the Galaxy. Thanks to the high resolution of the Chandra X-ray Observatory, many faint X-ray sources have been detected in this cluster (e.g. Heinke et al. 2006) when no transient was in outburst. When in outburst, a bright transient makes studying these faint sources very difficult and, in fact, frequent X-ray outbursts have been observed from Terzan 5. Chan$d r a$ observations during these outbursts allowed for accurate positions of the associated transients (Heinke et al. 2003 Pooley et al. 2010, 2011, Homan \& Pooley 2012) and so far three distinct X-ray transients have been identified in Terzan 5 (all NS systems; see for example Table 1 of Degenaar \& Wijnands 2012 and Table 5 of Bahramian et al., 2014). The transient NS LMXB EXO 1745-248 has been identified as the system responsible for several of the episodes of high X-

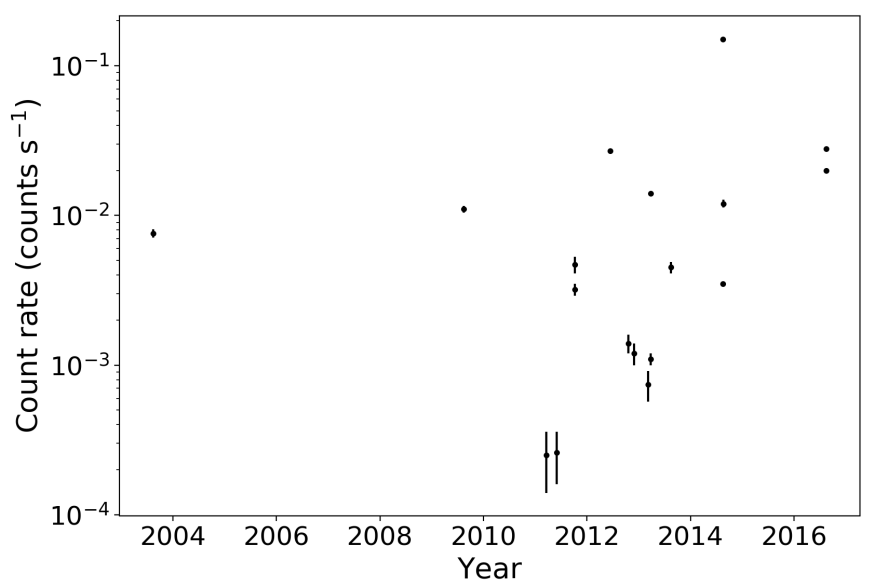

Figure 1. Light curve in the $0.5-10 \mathrm{keV}$ energy range of our Chandra observations of EXO 1745-248 during quiescence. The observations correspond to these described in Table 1

ray activity in Terzan 5 (see, e.g., Heinke et al. 2003 Serino et al. 2012, Degenaar \& Wijnands 2012; Wijnands et al. 2016 Tetarenko et al. 2016: Matranga et al. 2017).

The quiescent state of EXO 1745-248 has been investigated by Wijnands et al. (2005) and Degenaar \& Wijnands (2012) using Chandra observations of Terzan 5 when the transient was not in outburst. Both studies found that the quiescent X-ray spectrum of the source could be modeled with only a hard emission component with no need to add a soft component. In addition, Degenaar \& Wijnands (2012) found that the source was very variable between different observations; it exhibited a quiescent luminosity between $4 \times 10^{31} \mathrm{erg} \mathrm{s}^{-1}$ and $\sim 10^{33} \mathrm{erg} \mathrm{s}^{-1}$ during the period 2003-2011. In order to determine an upper limit on the contribution of a possible thermal component in the quiescent spectrum, Degenaar \& Wijnands (2012) added a NS atmosphere component to their power-law model. They determined a NS surface temperature of $\lesssim 42 \mathrm{eV}$. This low upper limit indicates that the NS in EXO $1745-248$ has a relatively cold core, suggesting that the NS efficiently cools in-between outbursts (for example through enhanced neutrino emission; Yakovlev \& Pethick 2004, Degenaar \& Wijnands 2012).

In this paper, we further study the quiescent state of EXO 1745-248. We complement the Chandra observations used by Wijnands et al. (2005) and Degenaar \& Wijnands (2012) with more Chandra data taken during the period $2011-2016$.

\section{OBSERVATIONS AND DATA REDUCTION}

To study the spectra and variability of the NS LMXB EXO 1745-248, we used data of the Galactic GC Terzan 5 obtained with Chandra. The data were taken at different epochs during the period 2003-2016 (see Table 1 for a $\log$ of the observation: ${ }^{1}$. The data were downloaded from

1 Besides the Chandra observations used in our paper, several additional Chandra pointings have been performed on Terzan 5 . However, during these observations one of the three transients located in this GC was active and their bright luminosities strongly 

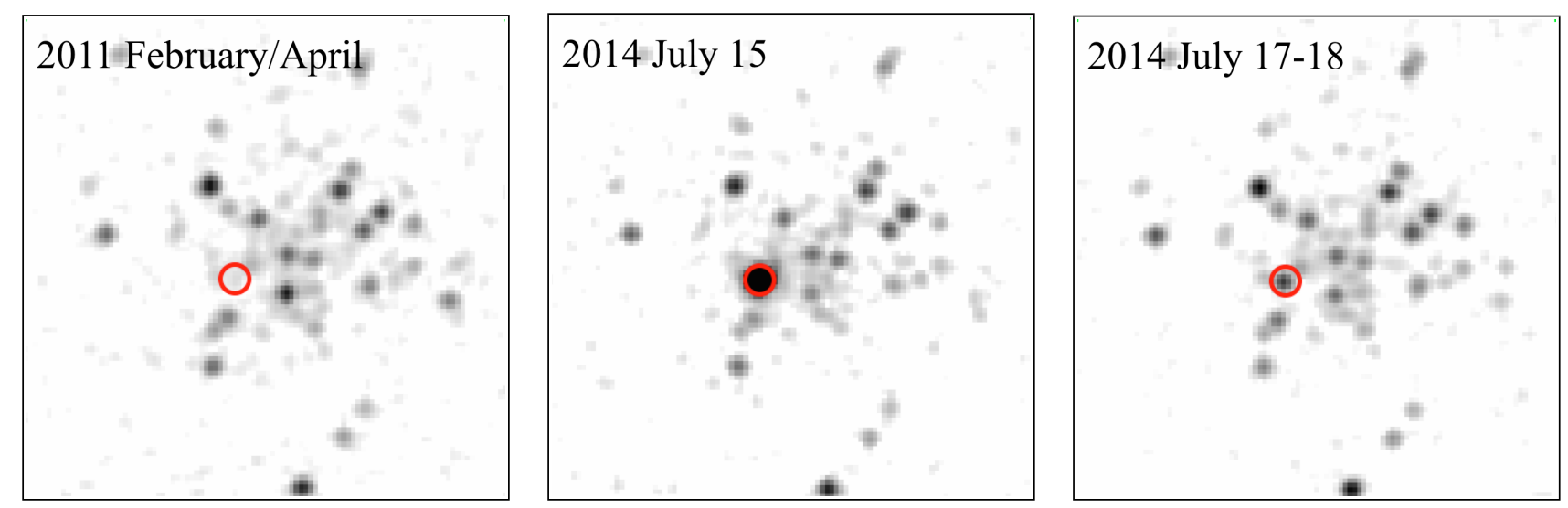

Figure 2. Chandra images of Terzan 5 in the $0.5-7 \mathrm{keV}$ energy range. The NS LMXB EXO $1745-248$ is indicated with a red circle. We show the data obtained during observations in which the source was the faintest (left panel; Feb-Apr 2011) and the brightest (middle panel; July 15, 2014). We also show in the right panel of this figure that only a few days after the July 15th, 2014, observation, the source was considerably fainter again, demonstrating that the source was highly variable on time scales of days (and not only on time scales of years). The image of 2011 is a stacked image of observations with IDs 13225 and 13252 . Note the large variability of the source between different epochs. Each image is $50^{\prime \prime} \times 50^{\prime \prime}$.

the Chandra archive ${ }^{2}$ All the data were taken in the faint mode, with a nominal frame time of $3.2 \mathrm{~s}$. The target was positioned on the S3 chip.

For the data reduction we have followed the Chandra threads ${ }^{3}$ which make use of the CIAO software package (v4.9; Fruscione et al. 2006). We recalibrated all the data using the chandra_repro script to assure that the newest calibrations were applied to the data.

To search for potential periods of high background (likely due to background flares), for each observation we created background light curves excluding the source region. No episodes of high background rates were observed for all but one observation: the data set with identification number (ObsID) 3798. Therefore, we reprocessed that data set following the appropriate Chandra tools ${ }^{4}$ to remove episodes of high background (i.e., the last $8.1 \mathrm{ks}$ of the observation were removed). This reduced its exposure time to $31.2 \mathrm{ks}$. The exposure times for all used observations are given in Table 1 .

In order to allow the X-ray data obtained during different observations to be stacked (e.g. to create the left panel of Figure 2, we carried out relative astrometry (besides the absolute astrometry provided by Chandra). To this end, we followed the appropriate Chandra thread ${ }^{5}$ and we created a broad band (0.5-7 keV) source catalog with the routine FLUXIMAGE. The corresponding PSF map was created with MKPSFMAP, while the WAVDETECT algorithm was used to locate the sources. The task wcs_match was used to perform a source cross match. This routine also determines the transformation parameters to shift a given image to the reference image (ObsID 15615).

The position of EXO 1745-248 has been previously constrained using Chandra by identifying the source in out-

influenced the data quality for faint sources. Therefore, we do not discuss these observations in this paper.

2 http://cxc.harvard.edu/cda/

3 http://cxc.harvard.edu/ciao/threads/

${ }^{4}$ http://cxc.harvard.edu/ciao/threads/filter/

${ }^{5}$ http://cxc.harvard.edu/ciao/threads/reproject_aspect/
Table 1. Log of the Chandra observations of EXO 1745-248 used in this paper. The net, background-corrected count rate given in column 4 corresponds to the energy band $0.5-10 \mathrm{keV}$. The count rates are averages for the whole observation.

\begin{tabular}{lccl}
\hline Date & Obs ID & $\begin{array}{c}\text { Exposure } \\
\text { time }(\mathrm{ks})\end{array}$ & $\begin{array}{l}\text { Net count rate } \\
(\text { counts s }\end{array}$ \\
\hline $2003-07-13 / 14$ & 3798 & $31.2^{1}$ & $7.6 \pm 0.5 \times 10^{-3}$ \\
$2009-07-15 / 16$ & 10059 & 36.3 & $1.12 \pm 0.06 \times 10^{-2}$ \\
$2011-02-17$ & 13225 & 29.7 & $2.5 \pm 1.1 \times 10^{-4}$ \\
$2011-04-29 / 30$ & 13252 & 39.5 & $2.6 \pm 1.0 \times 10^{-4}$ \\
$2011-09-05$ & 13705 & 13.9 & $4.7 \pm 0.6 \times 10^{-3}$ \\
$2011-09-08$ & 14339 & 34.1 & $3.2 \pm 0.3 \times 10^{-3}$ \\
$2012-05-13 / 14$ & 13706 & 46.5 & $2.79 \pm 0.08 \times 10^{-2}$ \\
$2012-09-17 / 18$ & 14475 & 30.5 & $1.4 \pm 0.2 \times 10^{-3}$ \\
$2012-10-28$ & 14476 & 28.6 & $1.2 \pm 0.2 \times 10^{-3}$ \\
$2013-02-05$ & 14477 & 28.6 & $7.4 \pm 1.7 \times 10^{-4}$ \\
$2013-02-22$ & 14625 & 49.2 & $1.44 \pm 0.05 \times 10^{-2}$ \\
$2013-02-23 / 24$ & 15615 & 84.2 & $1.1 \pm 0.1 \times 10^{-3}$ \\
$2013-07-16 / 17$ & 14478 & 28.6 & $4.5 \pm 0.4 \times 10^{-3}$ \\
$2014-07-15$ & 14479 & 28.6 & $1.48 \pm 0.02 \times 10^{-1}$ \\
$2014-07-17 / 18$ & 16638 & 71.6 & $3.49 \pm 0.02 \times 10^{-3}$ \\
$2014-07-20$ & 15750 & 23 & $1.16 \pm 0.07 \times 10^{-2}$ \\
$2016-07-13 / 14$ & 17779 & 68.9 & $2.84 \pm 0.06 \times 10^{-2}$ \\
$2016-07-15 / 16$ & 18881 & 64.7 & $2.04 \pm 0.06 \times 10^{-2}$ \\
\hline
\end{tabular}

1 Effective exposure time after the subtraction of background flares. The original exposure time was $39.3 \mathrm{ks}$.

burst (Heinke et al. 2003), resulting in a sub-arcsecond precision position of our target. This allows us to straightforwardly identify the right quiescent counterpart among the other faint cluster sources (see Figure 2). To extract light curves and spectra of EXO 1745-248, we used circular regions with variable radius centered on the source. This extraction radius varied from $\sim 1^{\prime \prime}$ to $\sim 1.7^{\prime \prime}$ according to the apparent size of the source, since the source was very variable in its brightness (see Figure 2 and Section 3.1). The background spectra were extracted from a source free part of the CCD using a circular region with a radius of $10^{\prime \prime}$. We 
used the task DMEXTRACT for creating light curves in the energy range $0.5-7 \mathrm{keV}$. The time bins were optimized (see Table 22 to avoid as much as possible bins with zero counts detected.

The tool SPECEXTRACT was used to obtain the source and background spectra (using the same extraction regions as used for the light curve extraction) and to generate the ancillary response files and redistribution matrix files. The data were grouped to have at least 15 counts per bin for most of the observations. This allows the use of the $\chi^{2}$ minimization fitting technique. However, for data sets in which the source was very faint, we rebinned the data to have at least 1 count per bin. For these observations we used the background subtracted Cash statistics (W-statistics; Wachter et al. 1979) instead of $\chi^{2}$ (see Table 2). The spectra were grouped using the tool DMGROUP.

To carry out all the spectral fitting we used the software package XsPec (v12.9.1 Arnaud 1996). To model the hydrogen column density $\mathrm{N}_{H}$ we used the model tbnew_ga: 5 with WILM abundances (Wilms et al. 2000) and VERN crosssections (Verner et al. 1996). We fitted our spectra with a power-law model (PEGPWRLW). In one case (ObsID 14479; Section 3.2, we also used a two component model consisting of a power-law model plus a black-body model (BBODYRAD). The luminosities were calculated using a distance towards Terzan 5 of $5.5 \pm 0.9 \mathrm{kpc}$ (Ortolani et al. 2007). All errors quoted in the paper correspond to $90 \%$ confidence levels.

\section{RESULTS}

\subsection{Spectral analysis and variability}

To study the quiescent spectral behavior of EXO 1745-248 we analyzed the X-ray spectrum of the source at different epochs in the energy band $0.5-10 \mathrm{keV}$. Wijnands et al. (2005) and Degenaar \& Wijnands (2012) carried out similar spectral studies of EXO 1745-248 in quiescence using a sub-set of the observations we are presenting. They found that the spectra of the source can be well described by a power-law model. The inclusion of a thermal component did not improve the spectral fits for the observations reported by these authors.

Based on the studies of Wijnands et al. (2005) and Degenaar \& Wijnands (2012), we have also fitted an absorbed power-law model (PEGPWRLW in XSPEC) to our Chandra observations of EXO 1745-248. In this model the hydrogen column density $\left(N_{H}\right)$, the photon index $(\Gamma)$ and the X-ray flux $\left(F_{X}\right)$ are the model parameters. Initially we left all of them free to vary in the fits (for all the spectra; see Table 2 for the results of these fits). However, for several data sets that have a small number of net counts, we noted that the fit produced very large errors for $N_{H}$ and $\Gamma$ or that the fit did not converge. This indicates that the obtained data for these observations are not of enough quality to constrain the parameters of the model. In these cases we fixed the values of $N_{H}$ and $\Gamma$ to $1.4 \times 10^{22} \mathrm{~cm}^{-2}$ and 1.4 , respectively. These values correspond to the average $N_{H}$ and $\Gamma$ derived from the observations for which the quality of the spectra was good enough to obtain reasonably constrained parameter values

\footnotetext{
6 http://pulsar.sternwarte.uni-erlangen.de/wilms/research/tbabs/
}

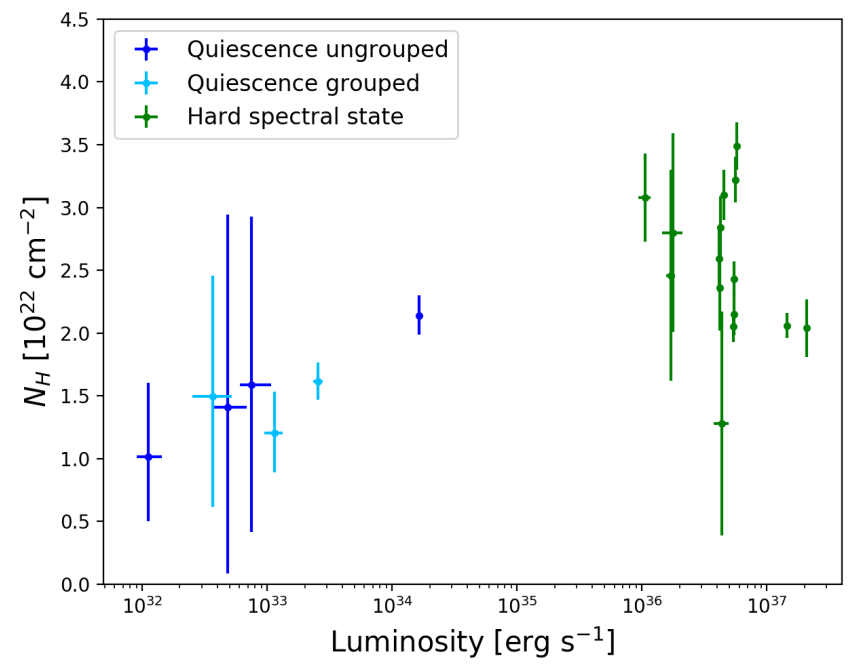

Figure 3. The hydrogen column density $\left(N_{H}\right)$ versus luminosity (0.5-10 keV) for EXO 1745-248 for the case in which $N_{H}, \Gamma$ and $F_{X}$ are free to vary for each individual spectrum. The blue (dark and light) points correspond to the quiescent state as analyzed in this work. Data (the light blue points) are grouped as in Table 2 The green points correspond to the very hard state data points of the source published by Parikh et al. (2017).

(these are the observations for which the $\chi^{2}$ statistics could be used). The obtained results are also shown in Table 2

For the observations performed in 2003 and 2009 we obtained values of the parameters that are consistent with those reported by Wijnands et al. (2005) and Degenaar \& Wijnands (2012). For the observations obtained in February and April of 2011 (ObsID 13225 and 13252), Degenaar \& Wijnands (2012) fixed the values of $N_{H}=1.2 \times 10^{22}$ $\mathrm{cm}^{-2}$ and $\Gamma=1.5$ and found a $0.5-10 \mathrm{keV}$ X-ray luminosity $L_{X} \sim 4 \times 10^{31} \mathrm{erg} \mathrm{s}^{-1}$. This value is consistent with our luminosities for the source during these observations, despite we have used different abundances and a slightly different $N_{H}$ absorption model.

We noted that for several observations similar values of $\Gamma$ were obtained at roughly similar X-ray luminosities (see Table 2). To improve on the obtained spectral parameters, we therefore combined several of these observations into groups. For each group we tied the parameters $N_{H}$ and $\Gamma$ but we left the fluxes free to vary since they were found to be not exactly the same when fitting the observations individually. We then fitted each group to obtain the associated spectral parameters. The results of these fits are also displayed in Table 2

In Figure 3 we show our obtained $N_{H}$ values in comparison with those obtained by Parikh et al. (2017) when EXO 1745-248 was observed at higher luminosities $\left(>1 \times 10^{36}\right.$ erg $\mathrm{s}^{-1}$ ) during the 2015 outburst of the source compared to our quiescent measurements (also shown in that figure). From this figure it can be seen that the $N_{H}$ during outburst reached higher values (although not always) than what we observe in quiescence. In addition, our quiescent data indicate by themselves a tentative increase of $N_{H}$ with luminosity. This suggests that the absorption internal to the system increases.

In Figure 4 we show $\Gamma$ versus $L_{X}$. Besides our results, 


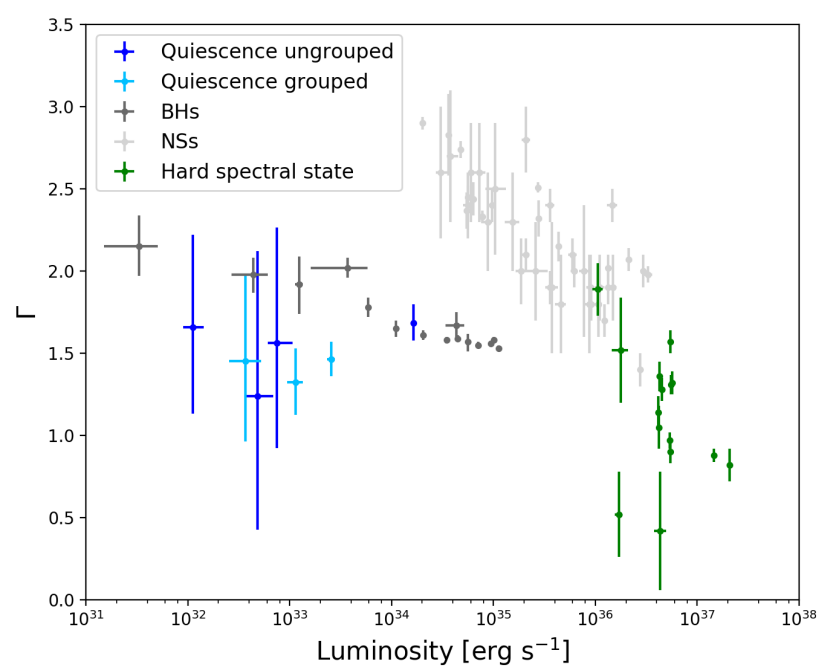

Figure 4. The photon index $\Gamma$ versus luminosity $(0.5-10 \mathrm{keV})$ for EXO 1745-248 in the case in which $N_{H}$ is allowed to vary for each individual spectra. The blue (dark and light) points are our quiescent points. The green points correspond to the very hard state identified for our source by Parikh et al. (2017). For comparison, the results for other NS systems (light gray points) and for BH systems (dark gray points) are also plotted (taken from Wijnands et al. 2015). The grouped light blue points correspond to the data groups given in Table 2

also the ones obtained by Parikh et al. (2017) during the 2015 outburst of the source and the data points presented in Figure 1 of Wijnands et al. (2015) for a large group of NS and $\mathrm{BH}$ systems. Clearly we see that in quiescence (below $1 \times 10^{34} \mathrm{erg} \mathrm{s}^{-1}$ ) EXO 1745-248 had consistently low photon indices of $\sim 1.5$ over the full 2 orders of magnitude range of the quiescence luminosities. The photon indices of our source were even lower (and thus the source had harder spectra) than BH systems at similar luminosities. Interestingly, the source had also rather low indices at luminosities above $1 \times 10^{36} \mathrm{erg} \mathrm{s}^{-1}$. These data points were indeed used by Parikh et al. (2017) to suggest that the source was in a newly identified very hard state during its 2015 outburst. Currently, we have no deep data for the luminosity range $\sim 1 \times 10^{34}$ to $\sim 1 \times 10^{36} \mathrm{erg} \mathrm{s}^{-1}$, so it is unclear if the source follows the general NS trend (and thus becomes softer below $1 \times 10^{36} \mathrm{erg} \mathrm{s}^{-1}$, but then suddenly hardens around $1 \times 10^{34}$ erg $\mathrm{s}^{-1}$, or that it stayed very hard over the full luminosity range covered in this figure).

In Figure 5 we show the spectra obtained for the observations with ObsIDs 14479, 13706, 14625 and 16638, taken during the period 2012-2014 where the strong variability of the object is clearly visible. The X-ray luminosity of EXO 1745-248 was found to vary from $4 \times 10^{32} \mathrm{erg} \mathrm{s}^{-1}$ up to $\sim 1.7 \times 10^{34} \mathrm{erg} \mathrm{s}^{-1}$ (see Table 2). This shows that during quiescence, the object exhibits luminosity variations of several orders of magnitude on time scales of days to years. This is also immediately visible in the different panels of Figure 2 and from the net count rate column in Table 1.

To investigate the variability of the source in more detail, we created light curves in the energy range $0.5-10 \mathrm{keV}$ (Figure 6). Due to the large difference in source count rates

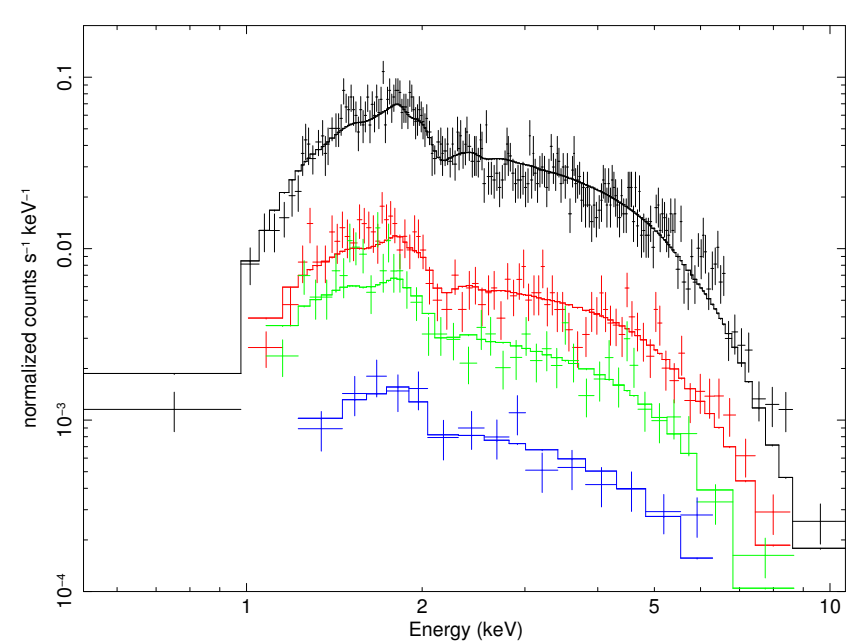

Figure 5. The Chandra spectra of EXO 1745-248 observed at different epochs during its quiescent state. The energy range is 0.5-10 keV. From top to bottom, the spectra obtained during the observations with the following ObsIDs are plotted: 14479,13706 , 14625 and 16638. In each of these observations the system shows luminosity variations of around two orders of magnitude in time scales of a few hours (see Figure 6). X-ray luminosities in the range $4 \times 10^{32}-1.7 \times 10^{34} \mathrm{erg} \mathrm{s}^{-1}$ are observed between these observations. The solid lines represent the best absorbed powerlaw model in which $N_{H}, \Gamma$ and $F_{X}$ were free parameters in the fit.

during the individual observations, we used a variety of time bin sizes (see Table 2) to highlight the variability, trying to avoid as much as possible time bins with count rates of zero. We do not plot the light curve corresponding to the data set with ObsID 13252 because of the very low number of counts detected during that observation.

We found that apart from the large count rate variations of the source between the various observations, the source also displays very strong short-term variability. This is shown in the light curves for observations with ObsIDs 13706, 14625 and 14479 (Fig. 6), in which variations of two orders of magnitude within a few hours are observed.

Although Figure 3 shows that $N_{H}$ might not be constant in quiescence (and thus not only due to interstellar absorption), it is also possible that $N_{H}$ might in fact remain constant. If the gas column is actually not changing, the inferred variability in $N_{H}$ would then indicate that the true intrinsic spectral shape of the source might change but be masked by the inferred $N_{H}$ variations. To investigate this, we refitted the spectra tying the $N_{H}$ between the individual observations. The parameters $\Gamma$ and $F_{X}$ were still left as free parameters for each spectrum. We only did this for the data for which we could use the $\chi^{2}$ statistics (i.e. we excluded the spectra with 1 count per bin, since they require the use of $\mathrm{W}$-statistics). The results of this analysis are given in Table 3 As can be seen from this table in comparison to the results in Table 2 the photon indices and fluxes are consistent for the individual observations. Therefore, it could be possible that $N_{H}$ remains constant in quiescence, but leaving it free would not significantly affect the obtained photon indices and fluxes (besides making the errors bars on the 

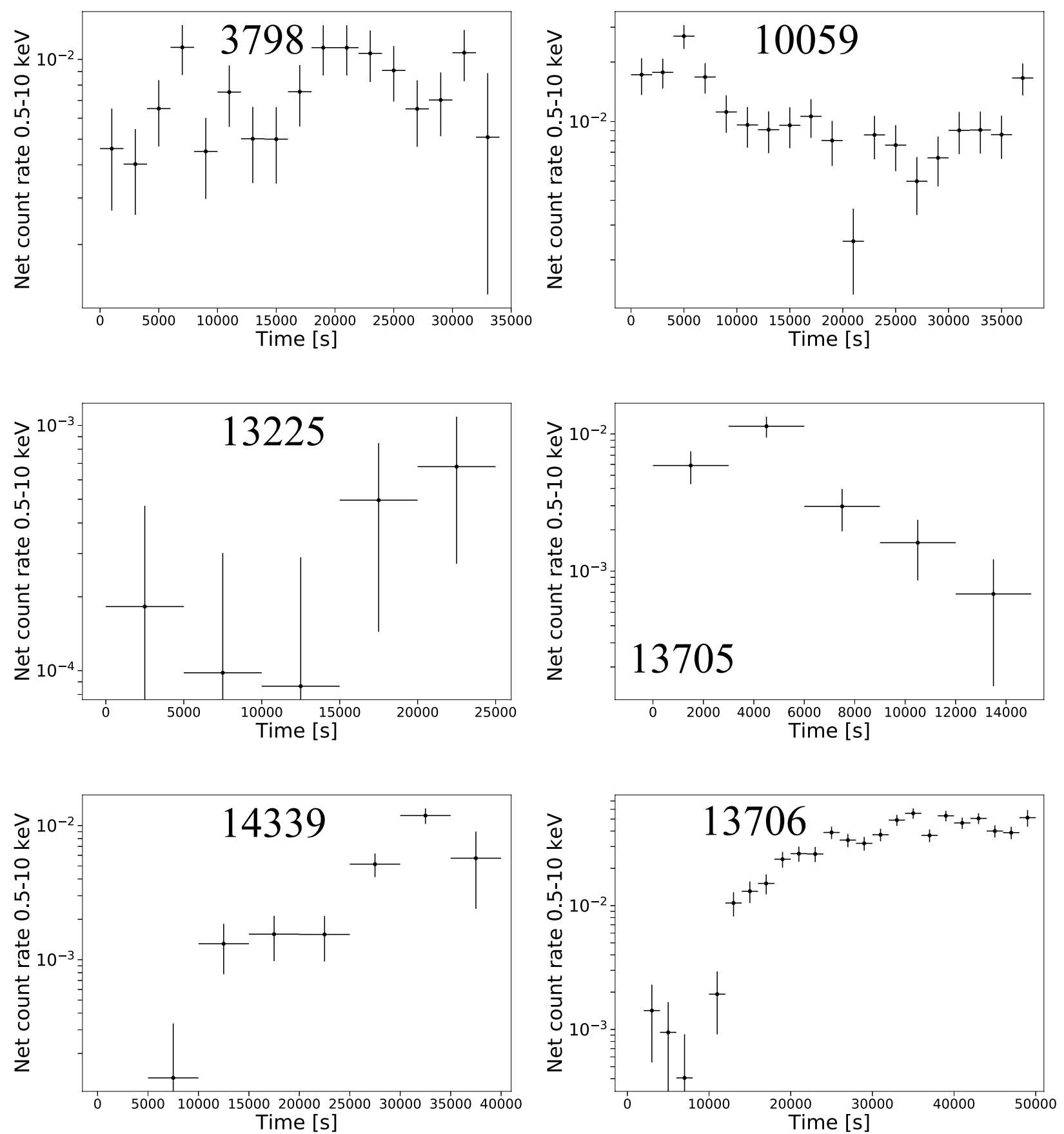

Figure 6. Light curves of EXO $1745-248$ in the $0.5-10 \mathrm{keV}$ X-ray band during quiescence. The ObsIDs correspond to the different Chandra observations listed in Table 1. Only the lightcurve for ObsID 13252 is not shown due to the small number of counts. For each ObsID we used a different bin size to highlight the variability but ensuring that we have as much as possible no bins with zero photons.

indices slightly larger). As in the case when we left also the $N_{H}$ as a free parameter for each observation, we grouped observations with similar $L_{X}$ and $\Gamma$. Thus, for each group of observations we also tied the value of $\Gamma$. We fitted the groups with the remaining individual observations (i.e. ObsIDs 3798 and 14479). Results for the groups are given in Table 3 (see also Figure 7 ). The values of $\Gamma$ and $F_{X}$ for the remaining individual observations are practically iden- tical to those obtained without grouping (keeping $N_{H}$ tied between observations) and therefore we do not show them.

\subsection{The thermal component of EXO 1745-248}

Given the high quality spectrum of ObsID 14479 (during which the source was brightest), and the unsatisfying fit obtained using an absorbed power-law model $\left(\chi_{\nu}^{2}=1.1\right.$ for 194 dof; p-value $p=0.14$ ), we added a thermal component 

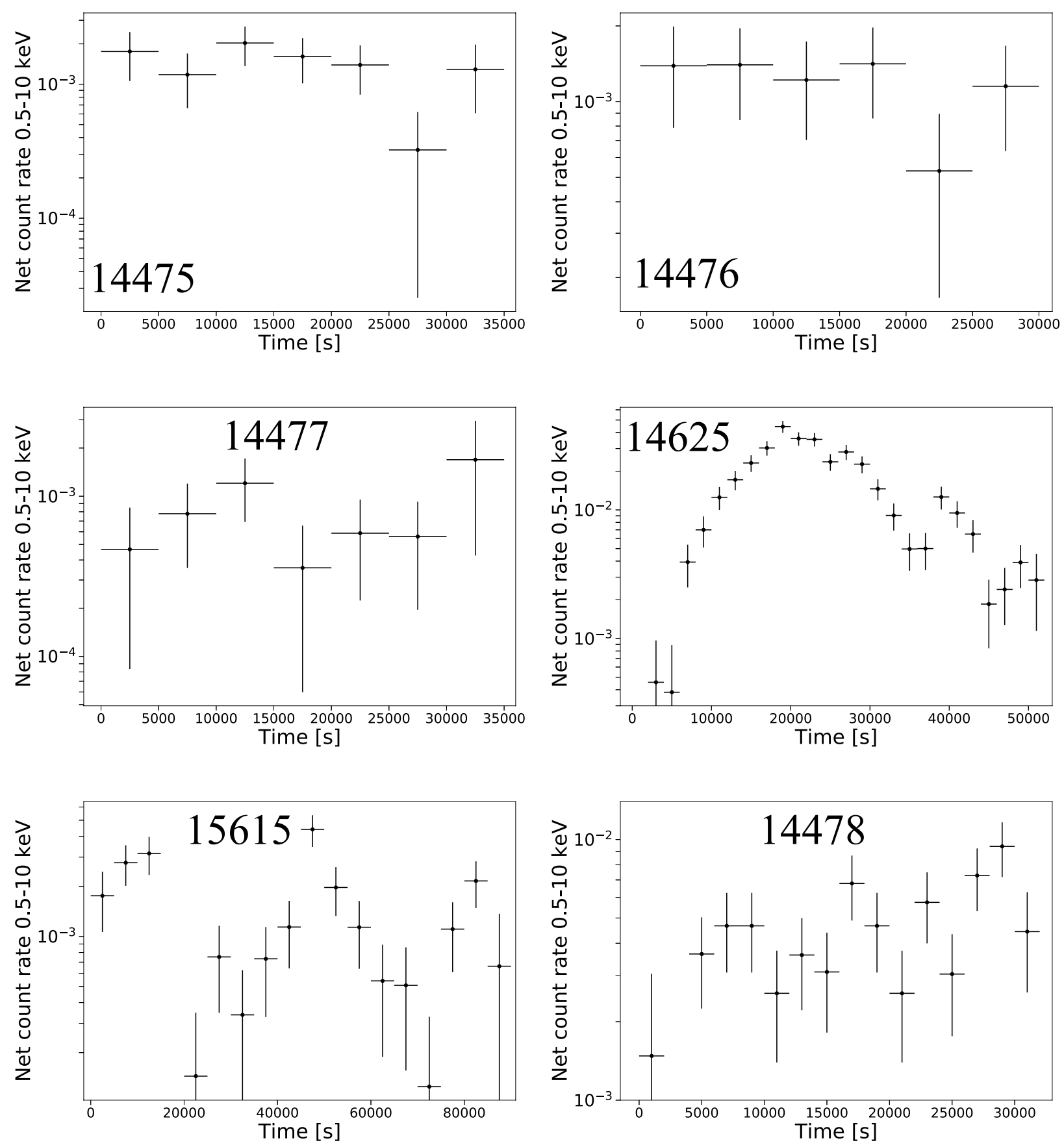

Figure 6. Continued

(black-body model, BBODYRAD in XSPEC). When using this two-component model assuming an emitting region with radius of $10 \mathrm{~km}$ at a distance of $5.5 \mathrm{kpc}$, we obtained the following parameters: $N_{H}=3.8 \pm 0.210^{22} \mathrm{~cm}^{-2}, \Gamma=1.5 \pm 0.1$, $F_{X}=45 \pm 2.0 \times 10^{-13} \mathrm{erg} \mathrm{cm}^{-2} \mathrm{~s}^{-1}$ and a temperature of $0.221 \pm 0.007 \mathrm{keV}$ for the black-body component. This fit produced a $\chi_{\nu}^{2}=0.9$ for 193 dof, with a $\mathrm{p}$-value $p=0.82$. This probability is large compared to that one obtained with the absorbed power-law model alone. When using this model, the power-law component contributed $\sim 41 \%$ of the total flux in the $0.5-10 \mathrm{keV}$ range. On the other hand, we found that the spectral fit for the second high quality spectrum (ObsID 17779) did not show substantial improvement when we added a thermal component. That spectrum is well described by a power-law model (Table 2).

In Figure 8, we show the spectrum (ObsID 14479) of EXO 1745-248 once more but now fitted with a powerlaw model (left panel) and with a two component model (right panel). The residuals are much smaller for the twocomponent model compared to the ones obtained when fitting a power-law model to the spectrum. An F-test between both models resulted in a probability $p \sim 3 \times 10^{-10}$ of ob- 

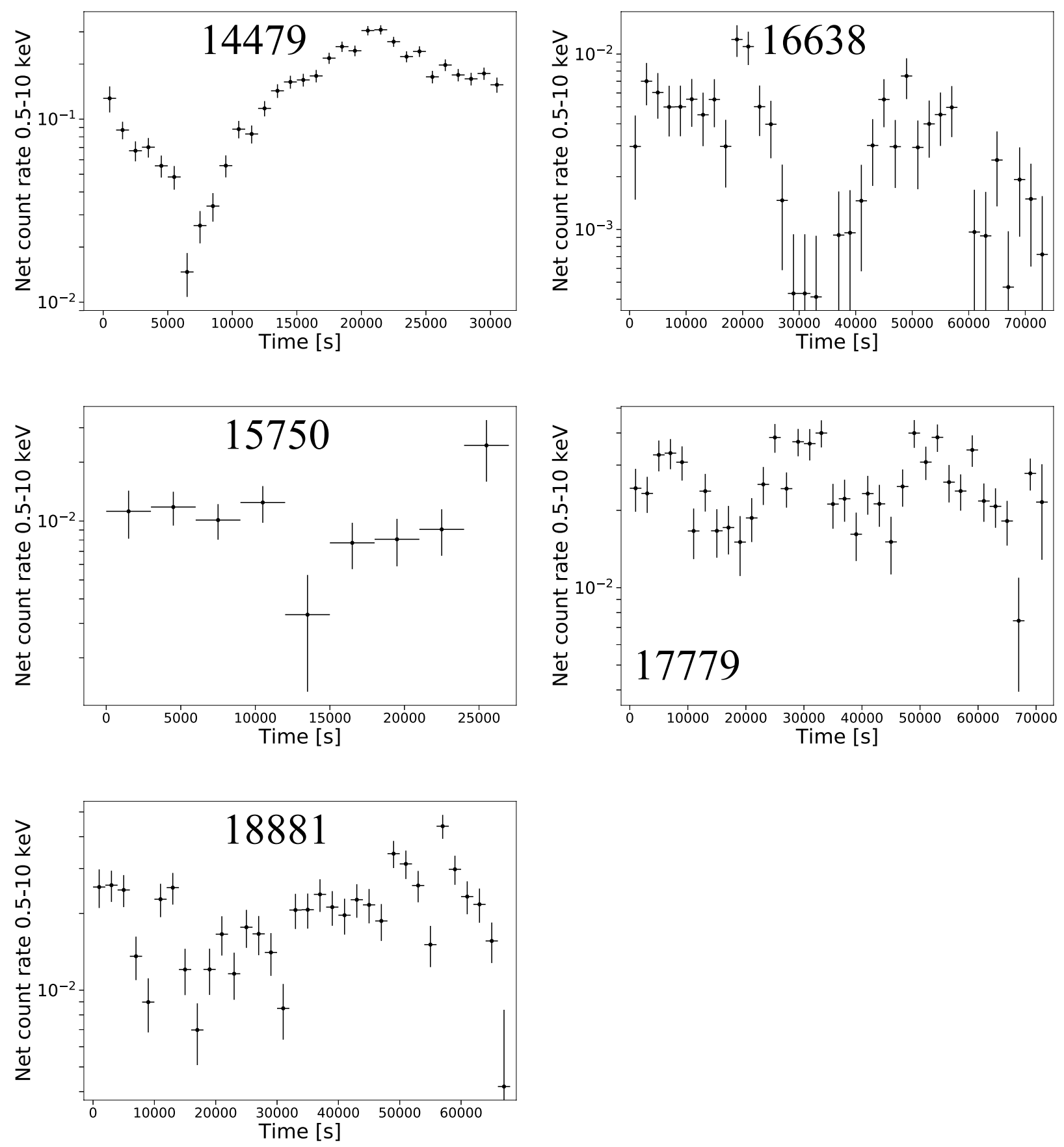

Figure 6. Continued

taining an improvement in the $\chi^{2}$ statistic by chance, as we found by adding a thermal component. Since this probability is $\ll 1$, it demonstrates that the two-component model describes the spectrum better than the single power-law model.

In the case in which we leave the radius of the thermal emitter free to vary, we obtain a $\mathrm{p}$-value $p=0.96$. In such case, the obtained parameter values are: $N_{H}=2.6 \pm 0.2$ $10^{22} \mathrm{~cm}^{-2}, \Gamma=1.0 \pm 0.1, F_{X}=38 \pm 1.5 \times 10^{-13} \mathrm{erg} \mathrm{cm}^{-2}$ $\mathrm{s}^{-1}$, a temperature of $0.34_{-0.03}^{+0.02} \mathrm{keV}$ and a radius of $2_{-1.0}^{+1.7}$ $\mathrm{km}$ for the thermal source. The F-test in this case gives a probability of $\sim 2 \times 10^{-13}$, again, suggesting that the data are better fitted by adding a black-body component. For this model, the power-law component contributed $\sim 64 \%$ of the total $0.5-10 \mathrm{keV}$ flux. Given the small radius obtained for the region of thermal emission, it is unlikely that it originates in an accretion disk.

We note that for the model in which we fix the radius of the black-body component, the obtained $N_{H}$ is very high $\left(\sim 3.8 \times 10^{22} \mathrm{~cm}^{-2}\right)$. This is even higher than what has been observed when the source was in outburst (Figure 3) and higher than the average value of $N_{H}$ for the cluster itself (Bahramian et al. 2015). But when leaving the radius as a 
Table 2. Results for the power-law model with $N_{H}, \Gamma$, and $F_{X}$ as free parameters (fitted in the energy range $0.5-10 \mathrm{keV}$ ). All quoted errors correspond to $90 \%$ confidence intervals. To calculate the luminosities $(0.5-10 \mathrm{keV})$, we assume a distance towards EXO $1745-248$ of $5.5 \mathrm{kpc}$. Group 1 corresponds to the observations with ObsID 14339, 14478 and 16638. Group 2 is formed by the observations with ObsID 10059, 14625 and 15750. Group 3 corresponds to the observations with ObsID 13706, 17779 and 18881 . When fitting the individual groups, the $N_{H}$ and $\Gamma$ were tied for each observation during the fitting, but the fluxes were left free. For each group, the X-ray fluxes and luminosities correspond to the average value of the individual fluxes. Column 8 indicates the length of the time bins used to create the light curves shown in Figure 6

\begin{tabular}{|c|c|c|c|c|c|c|c|}
\hline Date & ObsID & $\begin{array}{c}\mathrm{N}_{H} \\
\left(10^{22} \mathrm{~cm}^{-2}\right)\end{array}$ & $\Gamma$ & $\begin{array}{c}F_{X} \\
\left(10^{-13} \mathrm{erg} \mathrm{cm}^{-2} \mathrm{~s}^{-1}\right)\end{array}$ & $\begin{array}{c}L_{X} \\
\left(10^{33} \mathrm{erg} \mathrm{s}^{-1}\right)\end{array}$ & $\chi_{\nu}^{2}(\mathrm{dof})$ & $\begin{array}{l}\text { Time bin } \\
\text { size }(\mathrm{ks})\end{array}$ \\
\hline $2003-07-13 / 14$ & 3798 & $1.6_{-1.2}^{+1.3}$ & $1.5_{-0.6}^{+0.7}$ & $2.1_{-0.4}^{+0.9}$ & $0.7_{-0.1}^{+0.3}$ & $0.2(11)$ & 2 \\
\hline $2009-07-15 / 16$ & 10059 & $1.1_{-0.7}^{+0.8}$ & $1.0 \pm 0.4$ & $3.1 \pm 0.4$ & $1.1 \pm 0.1$ & $1.1(21)$ & 2 \\
\hline 2011-09-08 & 14339 & $<1.8$ & $0.9_{-0.4}^{+0.8}$ & $0.7 \pm 0.2$ & $0.3 \pm 0.1$ & $0.7(3)$ & 5 \\
\hline $2012-05-13 / 14$ & 13706 & $1.4_{-0.2}^{+0.3}$ & $1.2 \pm 0.2$ & $7.5 \pm 0.4$ & $2.7 \pm 0.2$ & $1.04(73)$ & 2 \\
\hline $2013-02-22$ & 14625 & $1.3 \pm 0.4$ & $1.5 \pm 0.3$ & $3.5_{-0.3}^{+0.4}$ & $1.3 \pm 0.1$ & $1.3(40)$ & 2 \\
\hline $2013-07-16 / 17$ & 14478 & $<4.5$ & $1.6_{-1.0}^{+1.4}$ & $1.2_{-0.3}^{+0.2}$ & $0.4_{-0.1}^{+0.9}$ & $0.9(4)$ & 2 \\
\hline $2014-07-15$ & 14479 & $2.1 \pm 0.1$ & $1.7 \pm 0.1$ & $45.5_{-1.9}^{+2.1}$ & $16.5_{-0.7}^{+0.8}$ & $1.1(194)$ & 1 \\
\hline $2014-07-17 / 18$ & 16638 & $1.7_{-1.1}^{+1.2}$ & $1.5_{-0.6}^{+0.7}$ & $1.0_{-0.2}^{+0.4}$ & $0.4 \pm 0.1$ & $0.7(12)$ & 2 \\
\hline $2014-07-20$ & 15750 & $1.1_{-1.0}^{+1.1}$ & $1.4_{-0.5}^{+0.6}$ & $2.8_{-0.4}^{+0.7}$ & $1.0_{-0.2}^{+0.3}$ & $1.6(13)$ & 3 \\
\hline $2016-07-13 / 14$ & 17779 & $1.7 \pm 0.2$ & $1.6_{-0.1}^{+0.2}$ & $8.0 \pm 0.5$ & $2.9 \pm 0.2$ & $1.04(104)$ & 2 \\
\hline \multirow[t]{5}{*}{$2016-07-15 / 16$} & 18881 & $1.6 \pm 0.3$ & $1.4 \pm 0.2$ & $5.7_{-0.3}^{+0.4}$ & $2.1 \pm 0.1$ & $1.3(74)$ & 2 \\
\hline & Group & $\begin{array}{c}\mathrm{N}_{H} \\
\left(10^{22} \mathrm{~cm}^{-2}\right)\end{array}$ & $\Gamma$ & $\begin{array}{c}\left\langle F_{X}\right\rangle \\
\left(10^{-13} \mathrm{erg} \mathrm{cm}^{-2} \mathrm{~s}^{-1}\right)\end{array}$ & $\begin{array}{c}\left\langle L_{X}\right\rangle \\
\left(10^{33} \mathrm{erg} \mathrm{s}^{-1}\right)\end{array}$ & & \\
\hline & 1 & $1.5 \pm 0.9$ & $1.4 \pm 0.5$ & $1.0_{-0.3}^{+0.4}$ & $0.4_{-0.1}^{+0.2}$ & $0.7(23)$ & \\
\hline & 2 & $1.2 \pm 0.3$ & $1.3 \pm 0.2$ & $3.1 \pm 0.5$ & $1.1 \pm 0.2$ & $1.3(78)$ & \\
\hline & 3 & $1.6 \pm 0.1$ & $1.5 \pm 0.1$ & $7.0 \pm 0.6$ & $2.6 \pm 0.2$ & $1.1(255)$ & \\
\hline Date & ObsID & $\begin{array}{c}\mathrm{N}_{H} \\
\left(10^{22} \mathrm{~cm}^{-2}\right)\end{array}$ & $\Gamma$ & $\begin{array}{c}F_{X} \\
\left(10^{-13} \mathrm{erg} \mathrm{cm}^{-2} \mathrm{~s}^{-1}\right)\end{array}$ & $\begin{array}{c}L_{X} \\
\left(10^{33} \mathrm{erg} \mathrm{s}^{-1}\right)\end{array}$ & W-stat (dof) & $\begin{array}{l}\text { Time bin } \\
\text { size }(\mathrm{ks})\end{array}$ \\
\hline $2013-02-23 / 24$ & 15615 & $1.0_{-0.5}^{+0.6}$ & $1.6_{-0.5}^{+0.6}$ & $0.3 \pm 0.1$ & $0.1 \pm 0.04$ & $1.2(89)$ & 5 \\
\hline $2011-02-17$ & 13225 & 1.4 fix & $1.4 \mathrm{fix}$ & $0.1_{-0.06}^{+0.09}$ & $0.04_{-0.02}^{+0.03}$ & $5.2(8)$ & 5 \\
\hline $2011-04-29 / 30$ & 13252 & 1.4 fix & $1.4 \mathrm{fix}$ & $0.08_{-0.04}^{+0.05}$ & $0.03_{-0.01}^{+0.02}$ & $16.6(11)$ & - \\
\hline 2011-09-05 & 13705 & $1.4_{-1.3}^{+1.5}$ & $1.2_{-0.8}^{+0.9}$ & $1.3_{-0.3}^{+0.64}$ & $0.5_{-0.1}^{+0.2}$ & $58.8(52)$ & 3 \\
\hline $2012-09-17 / 18$ & 14475 & $1.4 \mathrm{fix}$ & $1.4 \mathrm{fix}$ & $0.4 \pm 0.1$ & $0.1 \pm 0.04$ & $55.3(33)$ & 5 \\
\hline $2012-10-28$ & 14476 & $1.4 \mathrm{fix}$ & $1.4 \mathrm{fix}$ & $0.3_{-0.09}^{+0.1}$ & $0.1 \pm 0.04$ & $44.7(31)$ & 5 \\
\hline 2013-02-05 & 14477 & $1.4 \mathrm{fix}$ & $1.4 \mathrm{fix}$ & $0.2_{-0.07}^{+0.09}$ & $0.07_{-0.02}^{+0.03}$ & $21.3(19)$ & 5 \\
\hline
\end{tabular}

free parameter, the $N_{H}$ decreases to $2.6 \times 10^{22} \mathrm{~cm}^{-2}$ which is more in line with the other observations and with the average of the cluster (Bahramian et al. 2015), so this model might be more applicable. However, it is still unclear why the $N_{H}$ in this model is still significantly higher than observed for the other quiescent observations. This likely indicates correlated behaviour between the $N_{H}$ and the model parameters (i.e. the temperature and the photon index). However, it is important to note that despite adding a thermal component (which substantially improved the fit), the model shows residuals which suggest that a more complex model could improve even more the fit of the data. This in turn could explain the obtained high value of $N_{H}$.

\section{DISCUSSION}

We report on 18 Chandra observations (spanning from 2003 to 2016) performed on the GC Terzan 5 to study the quiescent properties of the transient NS LMXB EXO 1745-248. We found that the source is extremely variable: its quiescent luminosity (assuming a distance of $5.5 \mathrm{kpc} ; 0.5-10 \mathrm{keV}$ ) varies between $\sim 3 \times 10^{31} \mathrm{erg} \mathrm{s}^{-1}$ to $\sim 1.7 \times 10^{34} \mathrm{erg} \mathrm{s}^{-1}$ on time scales of days to years. Similarly to what was found previously by Wijnands et al. (2005) and Degenaar \& Wijnands (2012), we obtained quiescent spectra that are very hard, with typical photon indices of $\sim 1.4$ (when the spectra are fitted with a single power-law model). Intriguingly, the spectra do not significantly change over this large luminosity range (although the column density is potentially correlated with the luminosity ${ }^{7}$ Figure 3, except for the brightest observation during which a soft component needed to be added to obtain an acceptable fit (Section 3.2.

Wijnands et al. (2005) already reported on strong variability of the quiescent emission of EXO 1745-248. Also Degenaar \& Wijnands (2012) found that the source varied by a factor of $\sim 3$ within hours but by an order of magnitude between different observations (separated by years). Here we

7 As already suggested by Degenaar \& Wijnands (2012), this might be the reason why they found that the X-ray colors of the source during the 2003 observation (ObsID 3798) were softer than those obtained during the 2009 observation (ObsID 10059) despite that we have found that the photon indices during both observations are consistent with each other. 
Table 3. The fit results obtained in the $0.5-10 \mathrm{keV}$ band when tying $N_{H}$ between all spectra, but leaving $\Gamma$ and $F_{X}$ as free parameters. The resulting $N_{H}$ was $\mathrm{N}_{H}=1.8_{-0.9}^{+0.1} 10^{22} \mathrm{~cm}^{-2}$. All quoted errors correspond to $90 \%$ confidence intervals. The luminosities $(0.5-10 \mathrm{keV})$ were calculated using a distance towards EXO 1745-248 of $5.5 \mathrm{kpc}$. Only those observations for which the $\chi^{2}$ statistics could be used were considered for the fit. The resultant $\chi_{\nu}^{2}$ value was 1.15 for 565 dof for the individual observations and $\chi_{\nu}^{2}=1.17$ for 571 dof for the fit in which some of the individual spectra were grouped.

\begin{tabular}{lcccc}
\hline Date & ObsID & $\Gamma$ & $\begin{array}{c}F_{X} \\
\left(10^{-13} \mathrm{erg} \mathrm{cm}^{-2} \mathrm{~s}^{-1}\right)\end{array}$ & $\begin{array}{c}L_{X} \\
\left(10^{33} \mathrm{erg} \mathrm{s}^{-1}\right)\end{array}$ \\
\hline $2003-07-13 / 14$ & 3798 & $1.7 \pm 0.3$ & $2.1 \pm 0.2$ & $0.8 \pm 0.07$ \\
$2009-07-15 / 16$ & 10059 & $1.3 \pm 0.2$ & $3.4 \pm 0.3$ & $1.2 \pm 0.1$ \\
$2011-09-08$ & 14339 & $1.7_{-0.5}^{+0.6}$ & $0.9_{-0.1}^{+0.2}$ & $0.3_{-0.04}^{+0.07}$ \\
$2012-05-13 / 14$ & 13706 & $1.5 \pm 0.1$ & $7.8 \pm 0.4$ & $2.8 \pm 0.1$ \\
$2013-02-22$ & 14625 & $1.8 \pm 0.2$ & $3.9 \pm 0.3$ & $1.4 \pm 0.1$ \\
$2013-07-16 / 17$ & 14478 & $1.8 \pm 0.4$ & $1.3 \pm 0.2$ & $0.5 \pm 0.07$ \\
$2014-07-15$ & 14479 & $1.5 \pm 0.1$ & $43.0 \pm 1.4$ & $15.6 \pm 0.5$ \\
$2014-07-17 / 18$ & 16638 & $1.6 \pm 0.3$ & $1.0 \pm 0.1$ & $0.4 \pm 0.04$ \\
$2014-07-20$ & 15750 & $1.7 \pm 0.3$ & $3.2_{-0.3}^{+0.4}$ & $1.2 \pm 0.1$ \\
$2016-07-13 / 14$ & 17779 & $1.7 \pm 0.1$ & $8.3 \pm 0.4$ & $3.0 \pm 0.1$ \\
$2016-07-15 / 16$ & 18881 & $1.5 \pm 0.1$ & $5.9 \pm 0.3$ & $2.1 \pm 0.1$ \\
\hline & Group & $\Gamma$ & $\left\langle F_{X}\right\rangle$ & $\left\langle L_{X}\right\rangle$ \\
& & & $\left(10^{-13} \mathrm{erg} \mathrm{cm}^{-2} \mathrm{~s}^{-1}\right)$ & $\left(10^{33} \mathrm{erg} \mathrm{s}^{-1}\right)$ \\
\hline & 1 & $1.6 \pm 0.2$ & $1.1 \pm 0.3$ & $0.4 \pm 0.1$ \\
& 2 & $1.6 \pm 0.1$ & $3.4 \pm 0.5$ & $1.2 \pm 0.2$ \\
& 3 & $1.6 \pm 0.8$ & $7.3 \pm 0.6$ & $2.6 \pm 0.2$ \\
\hline
\end{tabular}

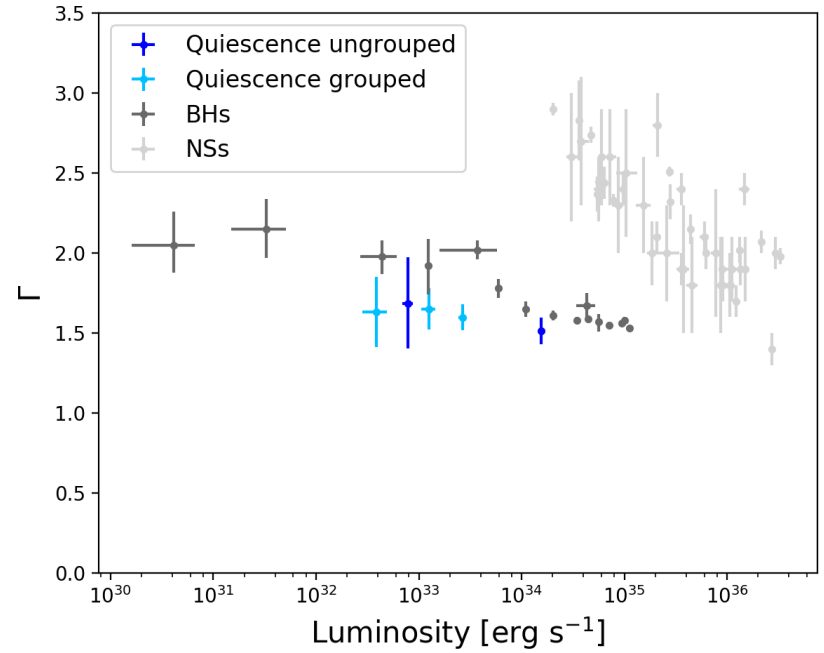

Figure 7. Similar to Figure 4 (without the very hard state points when the source was in outbursts; Parikh et al. 2017), but for the case in which $N_{H}$ is tied between the observations during the spectral fitting.

demonstrate that the variability of the source is even more extreme: its average luminosity varies by up to two orders of magnitude within $\sim 4$ hours (e.g., during observations with ObsID 13706, 14625 and 14479; Figure 6 to nearly three orders of magnitude on the timescale of years. Interestingly, the source was brightest during the observation performed on July 15th, 2014 (ObsID 14479), with an X-ray luminosity of $\sim 1.7 \times 10^{34} \mathrm{erg} \mathrm{s}^{-1}$. But only a few days later (on July 17th/18th, 2014; ObsID 16638), the source had decreased again down to $\sim 4 \times 10^{32} \mathrm{erg} \mathrm{s}^{-1}$. However, during both ob- servations the source was very variable as well (see Figure6) with a lowest count rate of only a few times $10^{-4}$ counts s ${ }^{-1}$ (ObsID 16638) and with a highest one of $\sim 3 \times 10^{-1}$ (ObsID 14479). This indicates count rate fluctuations in only three days of nearly three orders of magnitude.

Degenaar \& Wijnands (2012) discussed in detail the potential origin of the quiescent emission of EXO 1745-248 and concluded that the emission likely was due to very low level accretion down to the NS. However, recent observational insight (Cackett et al. 2010, Chakrabarty et al. 2014 D'Angelo et al. |2015; Wijnands et al.|2015) suggests that when low level accretion onto a NS occurs, the resulting Xray spectrum would have a soft thermal component (released when the matter hits the NS surface) as well as a hard, nonthermal one (due to Bremsstrahlung in the boundary layer). This is quite contrary to what we observe for EXO 1745248. Only during the brightest observation we need to add a soft component to obtain an acceptable fit. So, if indeed EXO 1745-248 is accreting at very low levels in its quiescent state, then (at least) below $10^{34} \mathrm{erg} \mathrm{s}^{-1}$ some other emission mechanism might be at work compared to the other quiescent NS LMXBs that are still accreting.

In this context, it is interesting to compare our source with the transient IGR J18245-2452 in the GC M28 ( $\mathrm{Pa}-$ pitto et al. 2013). This source is one of the so-called transitional millisecond pulsars (tMSPs), which are systems that transition between a radio millisecond pulsar phase and a phase in which they are accreting. IGR J18245-2452 is the only tMSP for which a bright $\left(\sim 10^{37} \mathrm{erg} \mathrm{s}^{-1}\right)$ outburst has been observed (Papitto et al. 2013, Linares et al. 2014, De Falco et al. 2017). During the outburst, the source showed several remarkable properties. Its spectra were always very hard (Linares et al. 2014 Parikh et al. 2017) and it showed very strong variability (Papitto et al.|2013 . De Falco et al. 2017. Wijnands et al. 2017). In addition, also its quiescent 
spectra are very hard and strongly variable (Linares et al. 2014). This, on first sight looks very similar to what we observed for EXO 1745-248. This NS LMXB was also very hard during (part of) its 2015 outburst (Tetarenko et al. 2016). Parikh et al. (2017) identified both sources as systems that exhibit a highly unusual very hard state at relatively high luminosities. Additionally, EXO 1745-248. was strongly variable during this phase (Wijnands et al. 2017). Similar strong variability was seen during the 2000 outburst of the source (Altamirano et al. in preparation), and its spectrum is also very hard and extremely variable in quiescence (see Section 3.1.

The extreme properties of IGR J18245-2452 have been attributed to the effect of the NS magnetic field in this system (see, e.g., the discussion in Ferrigno et al. 2014). This might suggest that something similar is possible for EXO 1745-248, and could explain why during our brightest quiescent observation (ObsID 14479) the source showed a thermal contribution potentially arising from only a small emitting region (see Section 3.2): the presence of a dynamically important magnetic field would potentially channel the accreted material only to the magnetic poles of the NS and not to the whole surface. This scenario would result in pulsations in the data of EXO 1745-248, but the time resolution of the ACIS detectors does not allow to search for them. However, detailed pulsation searches have been performed using the XMM-Newton observation obtained during the 2015 outburst of the source and none were found (upper limit in amplitude of 2\%; Matranga et al. 2017). Similarly, no pulsations were detected during the 2000 outburst of the source (Altamirano et al. in preparation) using the Proportional Counter Array on board of the Rossi X-ray Timing Explorer, which has a higher sensitivity to pulsations than XMM-Newton. Although weak pulsations might still be present for EXO 1745-248, it is unlikely that this system is, in this aspect, similar to IGR J18245-2452 (see also the discussion in Wijnands et al. 2017).

Another difference between IGR J18245-2452 and EXO $1745-248$ is the fact that the observed variability in IGR J18245-2452 was quite different during outburst (i.e., even more extreme) than observed for EXO 1745-248, potentially indicating different types of accretion flows (see Wijnands et al. 2017). On the other hand, during quiescence, the shortterm variability properties of EXO 1745-248 (Figure 6) are more extreme (and less regular) than what has been seen for IGR J18245-2452 (Linares et al. 2014). Finally, also the outflow properties are quite different: IGR J18245-2452 is a very strong (for a NS transient) radio source at its observed outburst luminosity $\left(\sim 10^{37} \mathrm{erg} \mathrm{s}^{-1}\right.$, Pavan et al. $2013 \mathrm{~Pa}-$ pitto et al. 2013), but EXO 1745-248 is actually the faintest NS radio source known at similar luminosities (for NS transients in the hard or very hard state; Tetarenko et al. 2016). This again suggests quite different accretion properties between both systems. So despite that the systems are both very hard over a very large luminosity range and show very strong variability, the details of their behavior differ. Thus, it is unclear whether we see the same accretion process active in both systems with only small changes between sources (such as the strength or configuration of the magnetic fields of the NSs) or that two, likely unrelated mechanisms are at work.

For EXO 1745-248 we have observed X-ray luminosities ranging up to $\sim 1.7 \times 10^{34} \mathrm{erg} \mathrm{s}^{-1}$ and the source is always very hard over the full luminosity range we explored. Parikh et al. (2017) report on Swift outburst data of this source (see also Tetarenko et al. 2016) and found that around $\sim 10^{36-37}$ erg $\mathrm{s}^{-1}$, EXO 1745-248 had also very hard spectra (it was one of the only three NS systems identified by these authors to have this previously unrecognised very hard state). The data reported by Parikh et al. (2017) only goes down to a luminosity of $\sim 10^{36} \mathrm{erg} \mathrm{s}^{-1}$ (see also Figure 4), with a potential minor softening at the lowest observed luminosity (albeit not very significant; only the lowest luminosity point showed a tentative softening). The spectral behavior of the source was unclear in the so far unexplored luminosity range of $10^{34}-10^{36} \mathrm{erg} \mathrm{s}^{-1}$. It might be possible that the source always remained hard and, in this case, the source would behave quite differently than what is observed for the majority of the NS systems (see Figure 4 and Figure 1 in Wijnands et al. 2015).

The softening of the other NS systems is thought to be due to the NS surface becoming dominantly present in the X-ray spectra (Wijnands et al. 2015). If for EXO 1745-248 the surface does not contribute equally strongly (in our preferred two component model the thermal component only contributes $\sim 36 \%$ to the $0.5-10 \mathrm{keV}$ flux instead of the usual $50 \%$ ), then the source indeed could remain hard over a very large luminosity range (from a few times $10^{31} \mathrm{erg}$ $\mathrm{s}^{-1}$ to $\sim 10^{37} \mathrm{erg} \mathrm{s}^{-1}$ ). In this case it is interesting to compare our source once again with IGR J18245-2452 because Linares et al. (2014, see also Parikh et al., 2017) found that this source indeed remained hard down to $\sim 10^{35} \mathrm{erg} \mathrm{s}^{-1}$, but softened (for unclear reasons) between $10^{34}$ and $10^{35}$ erg $\mathrm{s}^{-1}$. However, it became hard again at lower luminosities. Observations during a future outburst of EXO 1745-248 could test if a similar behavior is observed to that of IGR J18245-2452.

Alternatively, EXO 1745-248 might follow the same track as observed for the other NS systems (see Figure 4, but if true, at around $10^{34} \mathrm{erg} \mathrm{s}^{-1}$ it suddenly would have to become hard again (i.e., the NS would suddenly not be visible again; or only with a relatively small contribution). This would be roughly similar to the behavior of the accreting millisecond pulsar SAX J1808.4-3658 (Wijnands \& van der Klis 1998) which has a similar hard quiescent spectra (Campana et al. 2002, Heinke et al. 2007, 2009b), but above $10^{34} \mathrm{erg} \mathrm{s}^{-1}$ it follows the track of the other NS systems (see Figure 1 of Parikh et al. 2017). However, the quiescent luminosity of SAX J1808.4-3658 is very low, $\sim 8 \times 10^{31} \mathrm{erg} \mathrm{s}^{-1}$ and the transition from the soft spectra to the hard spectra below $10^{34} \mathrm{erg} \mathrm{s}^{-1}$ could be more gradual than what is required for EXO 1745-248. More detailed spectral observations are needed for all three sources (EXO 1745-248, IGR J18245-2452 and SAX J1808.4-3658) below $10^{36} \mathrm{erg} \mathrm{s}^{-1}$ to be able to make more conclusive statements about the similarities and differences between them, which would highlight potential differences in NSs and/or accretion properties.

\section{ACKNOWLEDGMENTS}

LERS acknowledges support from NOVA and a CONACyT (Mexico) fellowship. RW acknowledges support from a NWO top grant, Module 1. ND is supported by a Vidi grant 

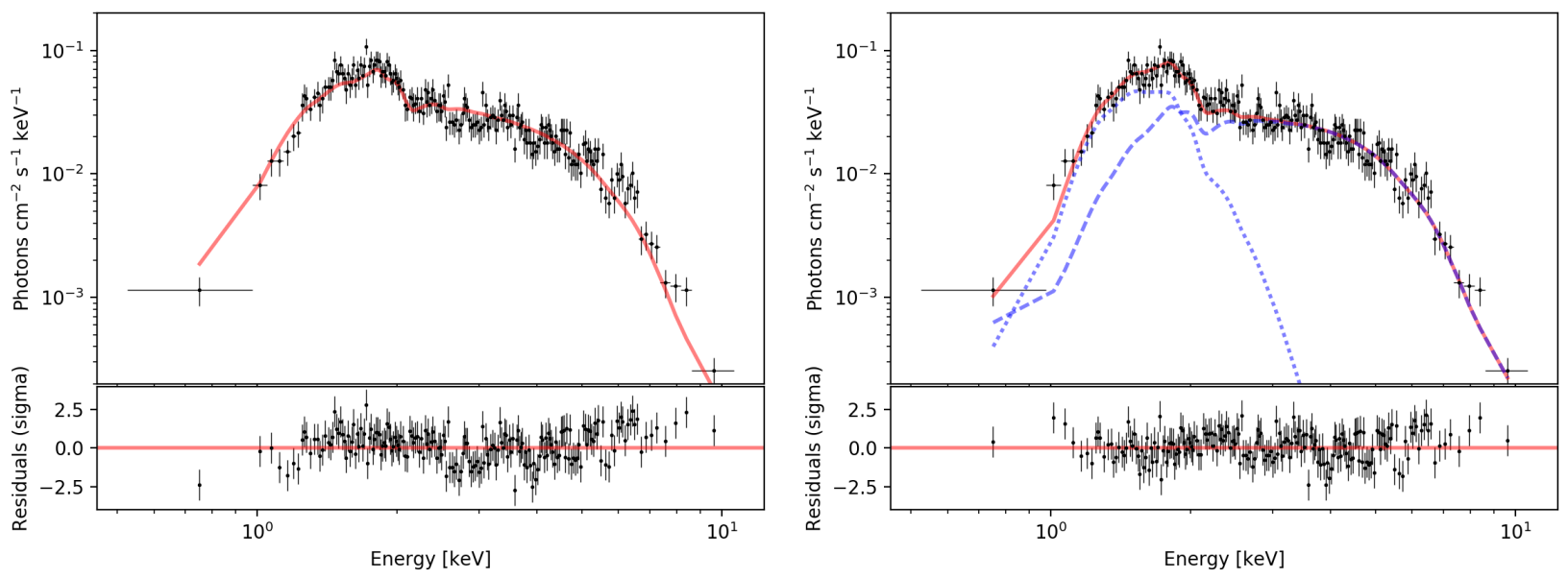

Figure 8. Comparison of the different models used to fit the spectra obtained for the observation with ObsID 14479. Left: A power-law model. Right: A model with a power-law plus a black-body component. The components are indicated with blue lines. The respective model is indicated with a solid red line. The residuals of each fit is indicated below in terms of sigma.

awarded by the NWO. YC is supported by the European Union Horizon 2020 research and innovation programme under the Marie Sklodowska-Curie Global Fellowship grant agreement No 703916. DA acknowledges support from the Royal Society. CH was supported by an NSERC Discovery Grant and Discovery Accelerator Supplement. The NASA ADS abstract service was used to access scientific publications and for getting the references used in this paper. Support for this work was provided by the NASA through Chandra Award Number GO6-17031B issued by the Chandra X-ray Observatory Center, which is operated by the Smithsonian Astrophysical Observatory for and on behalf of the NASA under contract NAS8-03060.

\section{REFERENCES}

Arnaud K. A., 1996, in Jacoby G. H., Barnes J., eds, Astronomical Data Analysis Software and Systems V Vol. 101 of Astronomical Society of the Pacific Conference Series, XSPEC: The First Ten Years. p. 17

Asai K., Dotani T., Hoshi R., Tanaka Y., Robinson C. R., Terada K., 1998, PASJ, 50, 611

Asai K., Dotani T., Mitsuda K., Hoshi R., Vaughan B., Tanaka Y., Inoue H., 1996, PASJ, 48, 257

Bahramian A., Heinke C. O., Degenaar N., Chomiuk L., Wijnands R., Strader J., Ho W. C. G., Pooley D., 2015, MNRAS, 452, 3475

Bahramian A., Heinke C. O., Sivakoff G. R., Altamirano D., Wijnands R., Homan J., Linares M., Pooley D., Degenaar N., Gladstone J. C., 2014, ApJ, 780, 127

Cackett E. M., Brown E. F., Miller J. M., Wijnands R., 2010, ApJ, 720, 1325

Cackett E. M., Wijnands R., Heinke C. O., Edmonds P. D., Lewin W. H. G., Pooley D., Grindlay J. E., Jonker P. G., Miller J. M., 2005, ApJ, 620, 922

Campana S., Colpi M., Mereghetti S., Stella L., Tavani M., 1998, A\&A Rev., 8, 279
Campana S., Stella L., Gastaldello F., Mereghetti S., Colpi M., Israel G. L., Burderi L., Di Salvo T., Robba R. N., 2002, ApJ, 575, L15

Chakrabarty D., Tomsick J. A., Grefenstette B. W., Psaltis D., Bachetti M., Barret D. e. a., 2014, ApJ, 797, 92

D'Angelo C. R., Fridriksson J. K., Messenger C., Patruno A., 2015, MNRAS, 449, 2803

De Falco V., Kuiper L., Bozzo E., Ferrigno C., Poutanen J., Stella L., Falanga M., 2017, A\&A, 603, A16

Degenaar N., Patruno A., Wijnands R., 2012a, ApJ, 756, 148

Degenaar N., Patruno A., Wijnands R., 2012b, ApJ, 756, 148

Degenaar N., Wijnands R., 2011, MNRAS, 412, L68

Degenaar N., Wijnands R., 2012, MNRAS, 422, 581

Ferrigno C., Bozzo E., Papitto A., Rea N., Pavan L., Campana S., Wieringa M., Filipović M., Falanga M., Stella L., 2014, A\&A, 567, A77

Fruscione A., McDowell J. C., Allen G. E., Brickhouse N. S., Burke D. J., Davis J. E. e. a., 2006, in Society of Photo-Optical Instrumentation Engineers (SPIE) Conference Series Vol. 6270 of SPIE Proc., CIAO: Chandra's data analysis system. p. $62701 \mathrm{~V}$

Hameury J.-M., Barret D., Lasota J.-P., McClintock J. E., Menou K., Motch C., Olive J.-F., Webb N., 2003, A\&A, 399,631

Heinke C. O., Edmonds P. D., Grindlay J. E., Lloyd D. A., Cohn H. N., Lugger P. M., 2003, ApJ, 590, 809

Heinke C. O., Jonker P. G., Wijnands R., Deloye C. J., Taam R. E., 2009a, ApJ, 691, 1035

Heinke C. O., Jonker P. G., Wijnands R., Deloye C. J., Taam R. E., 2009b, ApJ, 691, 1035

Heinke C. O., Jonker P. G., Wijnands R., Taam R. E., 2007, ApJ, 660, 1424

Heinke C. O., Wijnands R., Cohn H. N., Lugger P. M., Grindlay J. E., Pooley D., Lewin W. H. G., 2006, ApJ, 651, 1098

Homan J., Fridriksson J. K., Wijnands R., Cackett E. M., Degenaar N., Linares M., Lin D., Remillard R. A., 2014, 
ApJ, 795, 131

Homan J., Pooley D., 2012, The Astronomer's Telegram, 4302

in't Zand J. J. M., van Kerkwijk M. H., Pooley D., Verbunt

F., Wijnands R., Lewin W. H. G., 2001, ApJ, 563, L41

Kong A. K. H., McClintock J. E., Garcia M. R., Murray

S. S., Barret D., 2002, ApJ, 570, 277

Lasota J.-P., 2001, New A Rev., 45, 449

Linares M., Bahramian A., Heinke C., Wijnands R., Patruno A., Altamirano D., Homan J., Bogdanov S., Pooley D., 2014, MNRAS, 438, 251

Lowell A. W., Tomsick J. A., Heinke C. O., Bodaghee A., Boggs S. E., Kaaret P., Chaty S., Rodriguez J., Walter R., 2012, ApJ, 749, 111

Matranga M., Papitto A., Di Salvo T., Bozzo E., Torres D. F., Iaria R., Burderi L., Rea N., de Martino D., Sanchez-Fernandez C., Gambino A. F., Ferrigno C., Stella L., 2017, A\&A, 603, A39

Ortolani S., Barbuy B., Bica E., Zoccali M., Renzini A., 2007, A\&A, 470, 1043

Papitto A., Ferrigno C., Bozzo E., Rea N., Pavan L., Burderi L., Burgay M., Campana S., di Salvo T., Falanga 2013, Nature, 501, 517

Parikh A. S., Wijnands R., Degenaar N., Altamirano D., Patruno A., Gusinskaia N. V., Hessels J. W. T., 2017, MNRAS, 468, 3979

Pavan L., Wong G., Wieringa M. H., Tothill N. F. H., Filipovic M. D., Bozzo E., Ferrigno C., Papitto A., Romano P., 2013, The Astronomer's Telegram, 4981

Plotkin R. M., Gallo E., Jonker P. G., 2013, ApJ, 773, 59

Pooley D., Homan J., Altamirano D., Degenaar N., Heinke C. O., Lewin W., Sivakoff G. R., Wijnands R., 2011, The Astronomer's Telegram, 3743

Pooley D., Homan J., Heinke C., Linares M., Altamirano D., Lewin W., 2010, The Astronomer's Telegram, 2974

Rutledge R. E., Bildsten L., Brown E. F., Pavlov G. G., Zavlin V. E., 2001, ApJ, 551, 921

Rutledge R. E., Bildsten L., Brown E. F., Pavlov G. G., Zavlin V. E., 2002, ApJ, 577, 346

Serino M., Mihara T., Matsuoka M., Nakahira S., Sugizaki M., Ueda Y., Kawai N., Ueno S., 2012, PASJ, 64, 91

Tetarenko A. J., Bahramian A., Sivakoff G. R., Tremou E., Linares M., Tudor V., Miller-Jones J. C. A., Heinke C. O., Chomiuk L., Strader J., Altamirano D., Degenaar N., Maccarone T., Patruno A., Sanna A., Wijnands R., 2016, MNRAS, 460, 345

Tomsick J. A., Corbel S., Fender R., Miller J. M., Orosz J. A., Rupen M. P., Tzioumis T., Wijnands R., Kaaret P., 2003, ApJ, 597, L133

Tomsick J. A., Gelino D. M., Halpern J. P., Kaaret P., 2004, ApJ, 610, 933

Verner D. A., Ferland G. J., Korista K. T., Yakovlev D. G., 1996, ApJ, 465, 487

Wachter K., Leach R., Kellogg E., 1979, ApJ, 230, 274

Wijnands R., Bahramian A., Altamirano D., Sivakoff G., Heinke C., Degenaar N., 2016, The Astronomer's Telegram, 8982

Wijnands R., Degenaar N., Armas Padilla M., Altamirano D., Cavecchi Y., Linares M., Bahramian A., Heinke C. O., 2015, MNRAS, 454, 1371

Wijnands R., Degenaar N., Page D., 2017, in press

Wijnands R., Heinke C. O., Pooley D., Edmonds P. D.,
Lewin W. H. G., Grindlay J. E., Jonker P. G., Miller J. M., 2005, ApJ, 618, 883

Wijnands R., Homan J., Miller J. M., Lewin W. H. G., 2004, ApJ, 606, L61

Wijnands R., Nowak M., Miller J. M., Homan J., Wachter S., Lewin W. H. G., 2003, ApJ, 594, 952

Wijnands R., Parikh A. S., Altamirano D., Homan J., Degenaar N., 2017, ArXiv e-prints

Wijnands R., van der Klis M., 1998, Nature, 394, 344

Wilms J., Allen A., McCray R., 2000, ApJ, 542, 914

Yakovlev D. G., Pethick C. J., 2004, ARA\&A, 42, 169 